\title{
Prevalência de discinese escapular e dor no ombro em surfistas amadores do Rio Grande do Sul: um estudo transversal
}

\author{
Prevalence of scapular dyskinesis and shoulder pain in amateur surfers from Rio Grande do Sul: \\ A cross-sectional study
}

Prevalencia de discinese escapular y dolor de hombro en surfistas aficionados de Rio Grande de Sul: um estudio transversal

Bruna do Nascimento Gomes ${ }^{1}$, Maurício Scholl Schell ${ }^{2}$, Carolina Gomes Rosa ${ }^{3}$, Francisco Xavier de Araújo 4

RESUMO I O movimento de remada representa 51,4\% do tempo total de prática do surfe, gerando alta demanda muscular do complexo do ombro. Apesar disso, há uma lacuna na literatura sobre a prevalência de dor e discinese escapular (DE) em surfistas. Este estudo teve o objetivo de avaliar a prevalência de dor no ombro em surfistas amadores do estado do Rio Grande do Sul, no Brasil. Tratase de estudo observacional descritivo transversal. Foram incluídos 21 homens, com idade entre 18 e 42 anos, que praticassem surfe há no mínimo dois anos. Os desfechos avaliados foram DE estática, DE dinâmica, dor no ombro através da escala numérica da dor -, comprimento do músculo peitoral menor, e escore no The Western Ontario Shoulder Instability Index. As variáveis contínuas foram apresentadas em média e desvio-padrão. As variáveis categóricas foram expressas em percentual. Associações dos dados foram testadas através do teste qui-quadrado e do teste de correlação de Pearson. A DE estava presente em 71,4\% da amostra, tendo uma maior prevalência a discinesia do tipo I (57,1\%), e 42,9\% apresentaram dor no ombro durante o momento da avaliação. DE foi observada na maioria da população estudada, enquanto dor foi apresentada por pouco menos da metade dos participantes. Embora a DE seja um achado muito prevalente em surfistas amadores, não foi observada correlação com dor e redução da qualidade de vida. Descritores | Escápula/lesões; Dor; Ombro; Esporte.

ABSTRACT | The paddling movement represents $51.4 \%$ of total surfing practice time, generating high muscle demand for the shoulder complex. Despite this, there is a gap on the literature on the prevalence of pain and scapular dyskinesis (SD) in surfers. This study sought to evaluate the prevalence of SD and shoulder pain in amateur surfers in the state of Rio Grande do Sul, Brazil. It is a cross-sectional descriptive observational study. The sample consisted of 21 men, aged between 18 and 42 years, surfing for at least two years. The outcomes evaluated were static SD, dynamic SD, shoulder pain - by the numerical pain rating scale -, pectoralis minor muscle length, and score on the Western Ontario Shoulder Instability Index. Continuous variables were expressed in mean and standard deviation. Categorical variables were expressed as percentages. Data associations were tested through chi-square test and Pearson correlation test. SD was present in $71.4 \%$ of the sample, with a higher prevalence of Type I dyskinesia (57.1\%), and 42.9\% presented shoulder pain during evaluation. SD was observed in most of the studied population, while pain was present in just under half of the

Estudo realizado no Centro Universitário Ritter dos Reis (UniRitter) - Porto Alegre (RS), Brasil.

'Centro Universitário Ritter dos Reis (UniRitter) - Porto Alegre (RS), Brasil. E-mail: brunaangomes@gmail.com. Orcid: 0000-0001-9496-3075

2Universidade Federal de Ciências da Saúde de Porto Alegre (UFCSPA) - Porto Alegre (RS), Brasil. E-mail: mschell.fisio@gmail.com. Orcid: 0000-0002-3191-0360

3Universidade Federal de Ciências da Saúde de Porto Alegre (UFCSPA) - Porto Alegre (RS), Brasil. E-mail: gomesrosacarol@gmail.com. Orcid: 0000-0002-8419-197X

${ }^{4}$ Centro Universitário Ritter dos Reis (UniRitter) - Porto Alegre (RS), Brasil. E-mail: francisco araujo@uniritter.edu.br.

Orcid: 0000-0002-5637-4103 
participants. Although SD is a very prevalent find in amateur surfers, no correlation was observed between pain and reduced life quality. Keywords | Scapula/injuries; Pain; Shoulder; Sports.

RESUMEN | El movimiento del remo representa el 51,4\% del tiempo total de práctica de surf, generando una alta demanda muscular del complejo del hombro. Sin embargo, existe una brecha en la literatura sobre la prevalencia del dolor y la discinese escapular (DE) en los surfistas. Este estudio tuvo como objetivo evaluar la prevalencia de DE y dolor de hombro en surfistas aficionados del estado de Rio Grande do Sul, Brasil. Estudio observacional descriptivo transversal. Se incluyeron 21 hombres de 18 a 42 años que habían surfeando durante al menos 2 años. Los resultados evaluados fueron DE estática, DE dinámica, dolor de hombro usando la escala numérica de dolor, longitud del pectoral menor y puntaje en The Western Ontario Shoulder Instability Index. Las variables continuas se presentaron como media y desviación estándar. Las variables categóricas se expresaron como porcentaje. Las asociaciones de datos se probaron utilizando la Prueba Chi-cuadrado y el Coeficiente de Correlación de Pearson. La DE estaba presente en el $71,4 \%$ de la muestra, con una mayor prevalencia de discinesia tipo I (57,1\%) y el 42,9\% tenía dolor de hombro durante la evaluación. DE se observó en la mayoría de la población de estudio, mientras que el dolor en poco menos de la mitad de los participantes. Aunque el DE es un hallazgo muy frecuente en los surfistas, no hubo correlación con el dolor y la reducción de la calidad de vida.

Palabras clave | Escápula/lesiones; Dolor; Hombro; Deportes.

\section{INTRODUÇÃO}

O surfe é um esporte mundialmente conhecido estimando-se a existência de mais 35 milhões de surfistas em todo o mundo ${ }^{1}$. A Austrália, o Brasil e os Estados Unidos são considerados os países de maior potencial do surfe mundial ${ }^{2}$. No Brasil, estima-se que aproximadamente $1,3 \%$ da população (aproximadamente 1,9 milhões de pessoas) praticam o esporte ${ }^{3}$.

A remada, movimento de rotação interna e externa próximo de $90^{\circ}$ de abdução, é essencial para o surfe, pois é através dela que o atleta se desloca ${ }^{4}$, atingindo $51,4 \%$ do tempo total da prática no esporte ${ }^{5}$. O movimento repetitivo de ombro acima da cabeça e em alta velocidade durante a prática esportiva pode levar a uma descoordenação muscular no ombro ${ }^{5}$ essa, por sua vez, pode prejudicar os movimentos articulares ${ }^{6}$, levando a discinese escapular (DE).

DE corresponde a alterações biomecânicas em decorrência de desequilíbrios musculares, causando irregularidade na movimentação da escápula em relação à caixa torácica ${ }^{7}$. Alterações na cinemática escapular podem ocorrer devido à fadiga muscular, trauma, lesões préexistentes ${ }^{8}$, ou encurtamento do músculo peitoral menor 9 . O desequilíbrio muscular e alterações no sincronismo das articulações glenoumeral e escapulotorácica em praticantes de modalidades esportivas que exigem o movimento do membro superior acima da cabeça podem determinar condições dolorosas ${ }^{10}$. A prevalência da $\mathrm{DE}$ em outros esportes varia de $75 \%$ em jogadores de vôlei ${ }^{11}$ e $58,3 \%$ em nadadores. Sendo que, dentre esses atletas com DE, $80 \%$ têm dor no ombro $^{12}$. Além disso, a presença de pode aumentar em $43 \%$ o risco de dor no ombro, mesmo em atletas assintomáticos ${ }^{13}$.
Embora o surfe seja um esporte frequentemente praticado, não se sabe a prevalência de dor no ombro em praticantes do esporte. Portanto, o objetivo desse trabalho foi avaliar a prevalência de dor no ombro em surfistas amadores do estado do Rio Grande do Sul, no Brasil.

\section{METODOLOGIA}

Estudo observacional, descritivo, transversal, realizado de acordo com as recomendações Strobe ${ }^{14}$. O trabalho foi aprovado pelo Comitê de Ética e Pesquisa do Centro Universitário Ritter dos Reis (UniRitter) sob parecer $\mathrm{n}^{\circ}$ 2.945.304. Todos os participantes assinaram o termo de consentimento livre e esclarecido (TCLE).

Os participantes foram recrutados entre outubro a novembro de 2018. A amostragem ocorreu por conveniência através de anúncios digitais com a divulgação de imagem convite. Homens atletas amadores de surfe do Rio Grande do Sul, com idade entre 18 e 42 anos, que praticavam o esporte há no mínimo dois anos foram incluídos na pesquisa. Indivíduos com histórico de fratura no complexo do ombro, diagnóstico médico de capsulite adesiva, histórico de cirurgia no ombro, coluna cervical ou torácica e problemas neurológicos foram excluídos.

Um estudo piloto com 10 participantes foi realizado para análise da confiabilidade das medidas de avaliação. Os voluntários foram recrutados por conveniência e participaram do estudo piloto após a assinatura do TCLE.

Os sujeitos interessados em participar do estudo responderam um questionário online, para a verificação dos critérios de elegibilidade. O questionário era composto 
por perguntas sobre dados antropométricos, tempo de prática do surfe, tempo médio mensal de prática de surfe, presença de dor no ombro, histórico de luxação do ombro, fraturas, ou cirurgias e prática de outras atividades físicas.

Após o preenchimento do questionário, foi agendada coleta de dados presencial com os atletas aptos a participarem do estudo. As avaliações tiveram aproximadamente 20 minutos de duração. Todas as avaliações foram realizadas por uma mesma examinadora. A examinadora realizou um treinamento prévio com 10 indivíduos foi realizado para familiarização das avaliações.

A DE foi avaliada através dos métodos slide lateral scapular test (SLST) (avaliação estática) e filmagem (avaliação dinâmica). O SLST consiste na medida bilateral da distância entre o ângulo inferior da escápula e o processo espinhoso correspondente, com o ombro em três posições diferentes: $0^{\circ}, 45^{\circ}$ e $90^{\circ}$ de abdução ${ }^{15,16}$. Ao comparar os valores, assimetria maior que $1,5 \mathrm{~cm}$ entre os lados foi considerada presença de $\mathrm{DE}(\text { Figura } 1)^{8}$.

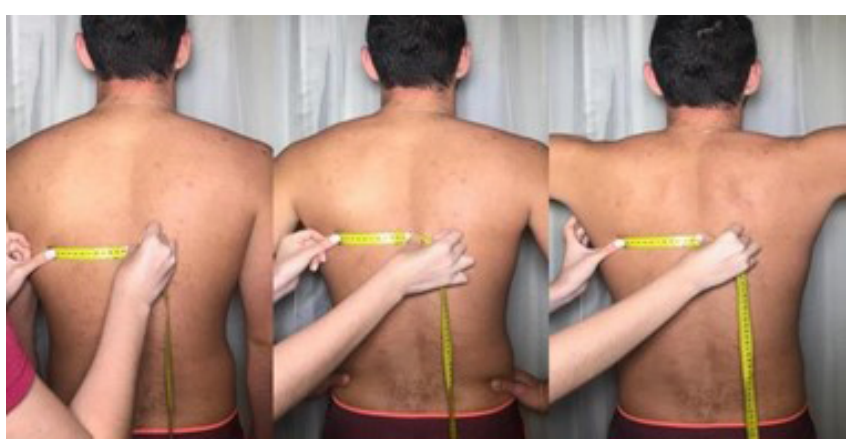

Figura 1. Teste SLST: distância entre o ângulo inferior da escápula e o processo espinhoso correspondente, posição neutra de membros superiores a $0^{\circ}$, abdução de ombro a $45^{\circ} \mathrm{e}$ abdução de ombro a $90^{\circ}$

Para a filmagem, o surfista permaneceu em ortostase, sem camisa e de costas para o avaliador. Foi posicionada uma câmera de celular a $204 \mathrm{~cm}$ de distância do atleta e com a altura variando de acordo com o indivíduo, de modo que a região escapular ficasse aparente. Foram realizadas três tentativas para familiarização com o movimento com três repetições ativas de elevação do ombro sem resistência ${ }^{17}$.A DE foi classificada em: (I) proeminência na borda medial da escápula; (II) a proeminência visualizada no ângulo inferior juntamente com a elevação escapular antecipada; (III) rotação escapular descendente e um rápido movimento escapular durante o retorno do braço ao lado do corpo; (IV) sem alteração ${ }^{18}$. Após, foi classificada em "sim" caso representasse os tipos I, II ou III e "não" caso representasse o tipo $\mathrm{IV}^{19}$.
A intensidade da dor no ombro no momento da coleta de dados foi avaliada através da escala numérica de dor (END) ${ }^{20}$. A END foi recomendada em estudos prévios baseados na facilidade de uso ${ }^{21}$, preferências do paciente ${ }^{22}$, menor inconsistência, poder discriminatório e reprodutibilidade ${ }^{23}$

O comprimento do peitoral menor foi mensurado com o indivíduo em ortostase, na posição neutra, relaxado e com os braços ao lado do corpo. A distância entre o processo coracóide e a $4^{\circ}$ articulação costoesternal ${ }^{9}$ foi medida com fita métrica (Figura 2) ${ }^{24}$. Este método foi validado e demonstrou-se confiável em comparação com paquímetro ${ }^{24}$. O índice do peitoral menor (IPM) foi calculado dividindo a medida do comprimento do músculo pela altura do indivíduo e multiplicando por 100. Foram considerados encurtados valores de PMI menores ou iguais a $7,65 \mathrm{~cm}^{25}$.

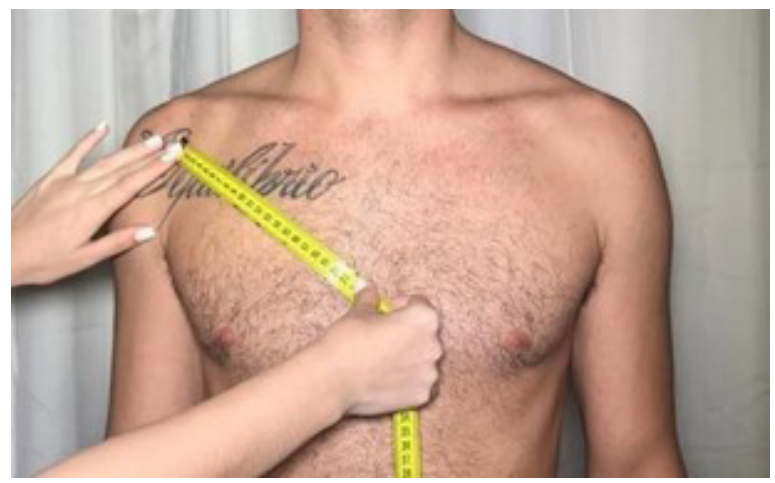

Figura 2. Medida do comprimento do músculo peitoral menor com uma fita métrica entre o processo inferomedial coracoide e a $4^{\circ}$ articulação costoesternal

A qualidade de vida foi avaliada através do The Western Ontario Shoulder Instability Index (WOSI) ${ }^{26}$. O WOSI, é um questionário designado para avaliar a qualidade de vida em pacientes com instabilidade no ombro, e é constituído por 21 questões, com quatro domínios: sintomas físicos; esportes, recreação e trabalho; estilo de vida e estado emocional. A pontuação obtida varia de 0 (nenhuma redução na qualidade de vida) a 2.100 pontos (pior qualidade devida) ${ }^{27}$.

Análises descritivas foram utilizadas para caracterizar a amostra. As variáveis contínuas foram testadas para normalidade com Shapiro-Wilk e apresentadas em média e desvio-padrão. As variáveis categóricas foram expressas em percentual. Associações entre as variáveis categóricas foram testadas através do teste qui-quadrado. Teste de correlação de Pearson foi usado para averiguar a associação de variáveis contínuas. Índice de correlação intraclasse (ICC) foi realizado para verificar a confiabilidade das 
medidas do estudo piloto. Os valores de significância de p $<0,05$ e Intervalo de Confiança de 95\% foram adotados.

\section{RESULTADOS}

Foram avaliados 21 atletas, suas características da estão apresentadas na Tabela 1.

Tabela 1. Características da amostra*

\begin{tabular}{|c|c|}
\hline Variável & $\mathrm{N}=21$ \\
\hline Idade & $28(5)$ \\
\hline Altura & $1,76(0,7)$ \\
\hline \multicolumn{2}{|l|}{ Dominância (\%) } \\
\hline $\mathrm{D}$ & 90,5 \\
\hline$E$ & 9,5 \\
\hline \multicolumn{2}{|l|}{ Lesão (\%) } \\
\hline Artrose & 4,8 \\
\hline Bursite & 4,8 \\
\hline Lesão de labrum & 4,8 \\
\hline Luxação & 14,3 \\
\hline Ruptura muscular & 4,8 \\
\hline Síndrome do impacto & 4,8 \\
\hline Tendinite & 14,3 \\
\hline Nenhuma & 47,6 \\
\hline \multicolumn{2}{|c|}{ Tempo de prática em horas/mês (\%) } \\
\hline 1 a $5 h$ & 14,3 \\
\hline 5 a $10 h$ & 23,8 \\
\hline 10 a $15 h$ & 23,8 \\
\hline 15 a $20 h$ & 19 \\
\hline+ de $20 \mathrm{~h}$ & 19 \\
\hline \multicolumn{2}{|c|}{ Tempo de prática em anos (\%) } \\
\hline 6 a 10 & 33,3 \\
\hline $10 \mathrm{ou}+$ & 66,7 \\
\hline \multicolumn{2}{|l|}{ Dor durante o surfe (\%) } \\
\hline Sim & 19 \\
\hline Não & 81 \\
\hline \multicolumn{2}{|l|}{ Dor após o surfe (\%) } \\
\hline Sim & 52,4 \\
\hline Não & 47,6 \\
\hline \multicolumn{2}{|c|}{ Prática de outra atividade física (\%) } \\
\hline Corrida & 19 \\
\hline Funcional & 47,6 \\
\hline Futebol & 28,6 \\
\hline Luta & 19 \\
\hline Musculação & 19 \\
\hline Natação & 33,3 \\
\hline Skate & 9,5 \\
\hline \multicolumn{2}{|c|}{ Frequência por semana (\%) } \\
\hline 1 & 5 \\
\hline 2 a 3 & 50 \\
\hline 3 a 4 & 20 \\
\hline $5 \mathrm{ou}+$ & 20 \\
\hline Nenhuma & 5 \\
\hline
\end{tabular}

*Valores expressos em média (desvio-padrão), exceto quanto apontado de outro modo.
O ICC realizado no estudo piloto, para avaliar a confiabilidade das medidas de DE e do comprimento do músculo peitoral menor, demonstraram boa confiabilidade entre as medidas, com valores de ICC entre 0,8 e 0,9.

A avaliação dinâmica da DE demonstrou que 15 indivíduos $(71,4 \%)$ apresentaram algum tipo $\mathrm{DE}$ no momento da coleta, tendo maior prevalência a $\mathrm{DE}$ do tipo I (57,1\%). A avaliação estática da DE demonstrou que 5 participantes $(23,8 \%)$ apresentaram resultado positivo. Nove atletas $(42,9 \%)$ apresentaram dor no ombro na avaliação presencial (Tabela 2). As respostas ao questionário WOSI ressaltaram um maior impacto na qualidade de vida devido a sintomas físicos, com uma média de 215,52 ( $\pm 171,74)$. O índice do peitoral menor não demonstrou valor correspondente a encurtamento muscular (Tabela 3).

Tabela 2. Discinese escapular avaliada da forma estática/dinâmica e dor no ombro

$\begin{array}{lr}\text { Variáveis } & \mathrm{n}=\mathbf{2 1} \\ \text { Discinese escapular - estática (\%) } & \\ \text { SLST } & 23,8 \\ \text { Positivo } & 76,2 \\ \text { Negativo } & \\ \text { Discinese escapular - dinâmica (\%) } & 57,1 \\ \text { Tipo I } & 14,3 \\ \text { Tipo II } & 0 \\ \text { Tipo III } & 28,6 \\ \text { Tipo IV } & 71,4 \\ \text { Positivo } & 28,6 \\ \text { Negativo } & \\ \text { Dor no ombro (\%), END } & 42,9 \\ \text { Sim } & 57,1 \\ \text { Não } & \end{array}$

END: escala numérica de dor; SLST: slide lateral scapular test.

Tabela 3. Questionário WOSI e índice do peitoral menor*

\begin{tabular}{lr} 
Variáveis & \multicolumn{1}{c}{$\mathrm{n}=\mathbf{2 1}$} \\
IPM & $10,93(0,96)$ \\
D & $11,40(1,01)$ \\
E & \\
WOSI & $215,52(171,74)$ \\
Sintomas físicos & $70,29(78,97)$ \\
Esporte, recreação e trabalho & $64,67(68,75)$ \\
Estilo de vida & $58,62(45,73)$ \\
Emoções &
\end{tabular}

*Valores expressos em média (desvio-padrão), exceto quanto apontado de outro modo. IPM: indice do peitoral menor; WOSI: The Western Ontario Shoulder Instability Index. 
Foi encontrada correlação baixa ( $V$ de Cramer 0,499) entre DE dinâmica e não praticar musculação (quiquadrado 5,219). Não houve correlação entre DE e dor no ombro; entre os métodos estático e dinâmico de avaliação da DE; entre dor, qualidade de vida e comprimento do peitoral menor; ou entre DE e comprimento do peitoral menor.

\section{DISCUSSÃO}

Este estudo teve como objetivo avaliar a prevalência de DE e dor no ombro em surfistas amadores. Foi observada prevalência de $71,4 \%$ de DE, avaliada dinamicamente, e $42,9 \%$ de dor no ombro nos indivíduos avaliados.

A presença de DE no âmbito esportivo é comum, principalmente em esportes que requerem movimentos amplos de membros superiores, que geram maior sobrecarga na musculatura do complexo articular do ombro, o que pode ocasionar dor no ombro ${ }^{11,12,28}$. Nesse sentido, o presente estudo apresenta resultados inovadores ao observar valores de prevalência de $\mathrm{DE}$ e dor em atletas praticantes de surfe, o que ainda não havia sido reportado na literatura. Os valores de ICC observados no estudo piloto variaram entre 0,8 e 0,9 , demonstrando boa confiabilidade entre as medidas de DE e comprimento do peitoral menor, o que reforça os achados deste estudo.

Apesar de ter sido observada a presença de DE e dor em atletas avaliados no presente estudo, não foi observada correlação entre os desfechos. Um estudo recente avaliou a DE através do SLST em 135 indivíduos separados em dois grupos, com e sem dor no ombro, e demonstrou que não houve correlação entre DE e dor no ombro, concluindo que a DE pode representar uma variabilidade normal do movimento ${ }^{29}$.

A correlação entre a presença de $\mathrm{DE}$ e a não realização de musculação encontrada neste estudo pode indicar que a prática dessa atividade física auxilia na redução do desequilibrio muscular do complexo do ombro. Neste sentido, detectar essa disfunção precocemente e realizar exercícios que possam reduzir esses desequilíbrios musculares pode ser uma ferramenta interessante na prevenção de lesões.

Diferentes resultados entre os métodos de avaliação de DE foram observados. A avaliação da forma dinâmica reflete melhor o gesto funcional do esporte. Ao comparar a DE durante um gesto concêntrico, excêntrico e em repouso, um estudo com 37 indivíduos praticantes de musculação observou que não há associação entre DE e dor no ombro ao repouso ou durante atividades de vida diária, porém, há relação entre $\mathrm{DE}$ e dor no ombro durante esforços. Indivíduos com $\mathrm{DE}$ apresentam 16 vezes mais chances de manifestar dor durante atividades que requerem maior esforço ${ }^{30}$. Estes achados corroboram com os nossos, uma vez que a avaliação do SLST na forma estática, em repouso, demonstrou que apenas $23,8 \%$ dos indivíduos apresentaram DE, e da forma dinâmica 71,4\% apresentaram o desfecho.

Sugere-se que o encurtamento do peitoral menor pode modificar o posicionamento da escápula ${ }^{9}$, entretanto, não encontramos nenhum indivíduo com encurtamento de peitoral menor. Da mesma forma, um estudo observou não haver diferença no comprimento do peitoral menor em sujeitos com e sem dor ${ }^{31}$.

Dentre os domínios do questionário WOSI, os indivíduos reportaram maiores reduções na qualidade de vida relacionadas aos sintomas físicos. Esse resultado pode demonstrar que a dor no ombro dos indivíduos avaliados os afeta durante a realização de determinados movimentos, corroborando com um estudo prévio envolvendo atletas de handebol ${ }^{32}$. Embora os atletas tenham apresentado $\mathrm{DE}$ e, alguns deles, dor, isso não parece ter influenciado em sua qualidade de vida e função, uma vez que os escores do questionário não foram altos.

Este estudo apresenta pontos fortes, como a população estudada e a boa confiabilidade das medidas realizadas. Porém, apresenta algumas limitações que devem ser consideradas. O número reduzido de participantes na pesquisa pode ter influenciado os resultados das análises, ainda que estudos semelhantes em atletas de vôlei e natação, avaliaram amostras de 12 e 36 sujeitos, respectivamente ${ }^{11,12}$. Além disso, foram coletados apenas atletas do sexo masculino e amadores, portanto, os resultados não podem ser extrapolados para outras populações.

\section{CONCLUSÃO}

Com o presente estudo, foi possível concluir que a $\mathrm{DE}$ foi observada na maioria da população estudada, e a dor em pouco menos da metade dos participantes. Embora a DE seja um achado muito prevalente em praticantes de surfe, não foi observada correlação com dor e redução da qualidade de vida. Futuros estudos longitudinais são necessários para verificar potenciais fatores de risco e métodos de tratamentos para essa população.

\section{REFERÊNCIAS}

1. Aguerre F. Olympic surfing. International Surfing Association [Internet]. 2008 Aug 20 [cited 2018 June 10]. Available from: http://www.isasurf.org/olympic-surfing/ 
2. Base LH, Alves MAF, Martins EO, Costa RF. Lesões em surfistas profissionais. Rev Bras Med Esporte. 2007;13(4):251-3. doi: 10.1590/S1517-86922007000400008

3. Brasil. Ministério do Esporte. Diagnóstico Nacional do Esporte (Diesporte). 2015 [cited 2018 May 20]. Available from: https:// edisciplinas.usp.br/pluginfile.php/376747/mod_resource/ content/1/Diesporte\%20-\%20MInist\%C3\%A9rio\%20do\% 20Esporte.pdf

4. Werner D. Surfer's start-up: a beginner's guide to surfing. 2nd ed. Ventura: Tracks Publishing; 1999.

5. Mendez-Villanueva A, Bishop DJ, Hamer PW. Activity profile of world-class professional surfers during competition: a case study. J Strength Cond Res. 2006;20(3):477-82. doi: 10.1519/16574.1

6. Amasay T, Karduna AR. Scapular kinematics in constrained and functional upper extremity movements. J Orthop Sports Phys Ther. 2009;39(8):618-27. doi: 10.2519/jospt.2009.2788

7. Phadke V, Camargo PR, Ludewig P. Scapular and rotator cuff muscle activity during arm elevation: a review of normal function and alterations with shoulder impingement. Rev Bras Fisioter. 2009;13(1):1-9. doi: 10.1590/s1413-35552009005000012

8. Kibler WB, McMullen JF. Scapular dyskinesis and its relation to shoulder pain. J Am Acad Orthop Surg. 2003;11(2):142-51. doi: 10.5435/00124635-200303000-00008

9. Borstad JD, Ludewig PM. The effect of long versus short pectoralis minor resting length on scapular kinematics in healthy individuals. J Orthop Sports Phys Ther. 2005;35(4):227-38. doi: 10.2519/jospt.2005.35.4.227

10. Ludewig PM, Braman JP. Shoulder impingement: biomechanical considerations in rehabilitation. Man Ther. 2011;16(1):33-9. doi: 10.1016/j.math.2010.08.004

11. Soliaman RR, Azzolini FL, Leme L, Ejnisman B, Pochini AC, Cunha RA. A influência do treinamento na discinesia escapular em jogadoras de voleibol: um estudo prospectivo. Rev Bras Med Esporte. 2015;21(3):206-9. doi: 10.1590/1517-869220152103141309

12. Santana EP, Ferreirar BC, Ribeiro G. Associação entre discinesia escapular e dor no ombro de praticantes de natação. Rev Bras Med Esporte. 2009;15(5):342-6. doi: 10.1590/ s1517-86922009000600004

13. Hickey D, Solvig V, Cavalheri V, Harrold M, Mckenna L. Scapular dyskinesis increases the risk of future shoulder pain by $43 \%$ in asymptomatic athletes: a systematic review and meta-analysis. $\mathrm{Br}$ J Sports Med. 2017;55(2):102-10. doi: 10.1136/bjsports-2017-097559

14. Malta M, Cardoso LO, Bastos FI, Magnanini MMF, Silva CMFP. Iniciativa Strobe: subsídios para a comunicação de estudos observacionais. Rev Saúde Pública. 2010;44(3):559-65. doi: 10.1590/s0034-89102010000300021

15. Kibler WB. The role of the scapula in athletic shoulder function. Am J Sports Med. 1998;26(2):325-37. doi: 10.1177/03635465980260022801

16. Taylor AB, Hurd CE, Deneger CR. Measurement of scapular asymmetry and assessment of shoulder dysfunction using the lateral scapular slide test: a reliability and validity study. Phys Ther. 2001;81(2):799-809. doi: 10.1093/ptj/81.2.799

17. Kibler WB, Uhl TL, Maddux JW, Brooks PV, Zeller BL, McMullen JF. Qualitative clinical evaluation of scapular dysfunction: A reliability study. J Shoulder Elbow Surg. 2002;11(6):550-6. doi: $10.1067 /$ mse.2002.126766
18. Kibler WB, Sciascia A. Current concepts: scapular dyskinesis. Br J Sports Med. 2009;44(5):300-5. doi: 10.1136/bjsm.2009.058834

19. Uhl T, Kibler WB, Gecewich B, Tripp BL. Evaluation of clinical assessment methods for scapular dyskinesis. Arthroscopy. 2009;25(11):1240-8. doi: 10.1016/j.arthro.2009.06.007

20. Hoggart B, Williamson A. Pain: a review of three commonly used pain rating scales. J Clin Nurs. 2005;14(7):798-804. doi: 10.1111/j.1365-2702.2005.01121.x

21. Paice JA, Cohen FL. Validity of a verbally administered numeric rating scale to measure cancer pain intensity. Cancer Nurs. 1997;20(2):88-93. doi: 10.1097/00002820-199704000-00002

22. Rodriguez CS, McMillan S, Yarandi H. Pain measurement in older adults with head and neck cancer and communication impairments. Cancer Nurs. 2004;27:425-33. doi: 10.1097/00002820-200411000-00001

23. Brunelli C, Zecca E, Martini C, Campa T, Fagnoni E, Bagnasco $\mathrm{M}$, et al. Comparison of numerical and verbal rating scales to measure pain exacerbations in patients with chronic cancer pain. Health Qual Life Outcomes. 2010;8:42. doi: 10.1186/1477-7525-8-42

24. Rosa DP, Borstad JD, Pires ED, Camargo PR. Reliability of measuring pectoralis minor muscle resting length in subjects with and without signs of shoulder impingement. Braz J Phys Ther. 2016;20(2):176-83. doi: 10.1590/bjpt-rbf.2014.0146

25. Struyf F, Mottram S, Roussel NA, Cools AMJ, Meeusen R. Clinical assessment of the scapula: a review of the literature. Br J Sports Med. 2012;48(11):883-90. doi: 10.1136/bjsports-2012-091059

26. Barbosa G, Leme L, Saccol MF, Pocchini A, Ejnisman B, Griffin S. Tradução e adaptação cultural para o português do Brasil do Western Ontario Shoulder Instability Index (WOSI). Rev Bras Med Esporte. 2012;18(1):35-7. doi: 10.1590/S1517-86922012000100007

27. Kirkley A, Griffin S, McLintock H, Ng L. The development and evaluation of a disease-specific quality of life measurement tool for shoulder instability. Am J Sports Med. 1998;26(6):764-72. doi: 10.1177/03635465980260060501

28. Silva RT, Hartmann LG, Laurino CFS, Biló JPR. Clinical and ultrasonographic correlation between scapular dyskinesia and subacromial space measurement among junior elite tennis players. Br J Sports Med. 2008;44(6):407-10. doi: 10.1136/bjsm.2008.046284

29. Plummer HA, Sum JC, Pozzi F, Varghese R, Michener LA. Observational scapular dyskinesis: known groups validity in patients with and without shoulder pain. J Orthop Sports Phys Ther. 2017;47(8):530-7. doi.org/10.2519/jospt.2017.7268

30. Mello AMS, Batista LSP, Oliveira VMA, Pitangui ACCR, Cattuzo MT. Associação entre discinese escapular e dor no ombro em praticantes de musculação. Rev Bras Ciênc Saúde. 2014;18(4):309-14. doi: 10.4034/rbcs.2014.18.04.05

31. Lewis JS, Valentine RE. The pectoralis minor length test: a study of the intra-rater reliability and diagnostic accuracy in subjects with and without shoulder symptoms. BMC Musculoskelet Disord. 2007;8(1):56-64. doi: 10.1186/1471-2474-8-64

32. Myklebust G, Hasslan L, Bahr R, Steffen K. High prevalence of shoulder pain among elite Norwegian female handball players. Scand J Med Sci Sports. 2011;23(3):288-94. doi: 10.1111/j.1600-0838.2011.01398.x 